\section{BMJ Open} Ophthalmology

\title{
Effect of first-line antifungal treatment on ocular complication risk in Candida or yeast blood stream infection
}

\author{
Nina Hautala (D) , , ${ }^{1,2}$ Hannu Köykkä, ${ }^{3}$ Mira Siiskonen, ${ }^{1,2}$ Juho Saari, ${ }^{2}$ Jari Kauranen, ${ }^{4}$ \\ Timo Hautala (1) ${ }^{3,5}$
}

To cite: Hautala N, Köykkä H, Siiskonen $\mathrm{M}$, et al. Effect of first-line antifungal treatment on ocular complication risk in Candida or yeast blood stream infection. BMJ Open Ophthalmology 2021;6:e000837. doi:10.1136/ bmjophth-2021-000837

Received 21 June 2021 Accepted 3 September 2021
Check for updates

(c) Author(s) (or their employer(s)) 2021. Re-use permitted under CC BY-NC. No commercial re-use. See rights and permissions. Published by BMJ.

${ }^{1}$ Department of Ophthalmology, Oulu University Hospital, Oulu, Finland

${ }^{2}$ PEDEG0 Research Unit and Medical Research Center, University of Oulu, Oulu, Finland ${ }^{3}$ Department of Internal Medicine, Oulu University Hospital, Oulu, Finland ${ }^{4}$ Nordlab, Oulu University Hospital, Oulu, Finland

${ }^{5}$ Research Unit of Biomedicine, University of Oulu, Oulu, Finland

Correspondence to DrTimo Hautala; timo.hautala@ oulu.fi

\section{ABSTRACT}

Objectives Ocular candidiasis $(\mathrm{OC})$ can complicate Candida bloodstream infection (BSI). Antifungal treatment improves the prognosis of patients with BSI, but the effects of choice and timing of first-line medication on $\mathrm{OC}$ risk are incompletely understood. We explored the early treatments, risk factors and ocular presentations in Candida BSI.

Methods and analysis All patients $(n=304)$ with Candida BSI during 2008-2017 at Oulu University Hospital were included. Those patients in whom clinical condition was appropriate for ocular examination (OE), including biomicroscopy $(n=103)$, were carefully analysed by ophthalmologists. Criteria for patient selection were considered. Candida and yeast species, antifungal medications, echocardiography, underlying diseases and clinical properties of the patients with Candida BSI were analysed.

Results Clinical condition in 103 patients had been considered appropriate for $\mathrm{OE}$. OC was diagnosed in 33 of the 103 patients. Candida albicans was the most common finding (88\%) in OC. Patients in intensive care, alcoholrelated conditions or poor prognosis were less frequently examined. Persistent candidemia increased the risk of $\mathrm{OC}$. Chorioretinitis and endophthalmitis were diagnosed in $94 \%$ and $48 \%$ of the patients with OC, respectively. Any early antifungal treatment decreased the endophthalmitis risk. Echinocandin lowered the $\mathrm{OC}$ risk in those with central venous catheters (CVCs) or abdominal malignancy. Conclusion Critical condition of patients with Candida $\mathrm{BSI}$ affects the selection and results of $\mathrm{OE}$. OC was associated with $C$. albicans BSI especially among those with persistent candidemia, CVC or abdominal malignancy. Any early antifungal treatment reduced endophthalmitis risk. Early echinocandin treatment may reduce the risk of $\mathrm{OC}$ in selected patients.

\section{INTRODUCTION}

Candida bloodstream infection (BSI) is commonly associated with ocular complications. Routine ophthalmological examination (OE) has been recommended for all patients with Candida BSI by the Infectious Diseases Society of America, ${ }^{1}$ although this practice remains controversial. ${ }^{2-5}$ Different definitions of probable or proven ocular candidiasis (OC) have led to variations in the conclusions of rate and outcomes of OC. ${ }^{36}$ Critical

\section{Key messages}

What is already known about this subject?

- Candida blood stream infection (BSI) may be complicated by ocular candidiasis (OC). Candida albicans is the most prevalent microbiological finding in $\mathrm{OC}$.

\section{What are the new findings?}

Our patient selection was carefully analysed and only those patients examined with biomicroscopy were included. Early antifungal treatment of Candida BSI reduced endophthalmitis risk. Early echinocandin treatment compared with fluconazole treatment reduced $\mathrm{OC}$ risk in patients with central venous catheter or abdominal malignancy.

How might these results change the focus of research or clinical practice?

- Early appropriate antifungal treatment can reduce risk of ocular complications in patients with Candida BSI. Early echinocandin treatment may reduce $\mathrm{OC}$ risk despite the low intraocular penetration.

condition of patients with Candida BSI may not warrant $\mathrm{OE}$ and all published data on $\mathrm{OC}$ are based on selected patient material. ${ }^{6}$

Candida BSI associates with serious underlying medical conditions and high mortality. ${ }^{1}$ Critically ill patients in intensive care units, those with major abdominal surgical procedures and patients with neutropenia are at highest risk. The patients frequently suffer from Candida colonisation of indwelling catheters, translocation from the gut to the bloodstream or anastomotic leakage after laparotomy. Candida endocarditis, that is, infectious process of heart, is a rare and serious complication with a high potential of affecting peripheral organs. $C$ albicans, C. glabrata, C. tropicalis, C. parapsilosis or C. krusei are the most common isolates in blood cultures although other Candida spp can be found. ${ }^{7} C$. albicans is the most common finding in OC. ${ }^{2-5}$ 
Echinocandins with broad-spectrum activity against Candida spp are commonly recommended for Candida BSI treatment. This group of antifungals diffuses poorly into the vitreous humour. ${ }^{18}$ Fluconazole, an agent active mainly against C. albicans, is more efficient in ocular penetration following systemic administration. Early appropriate antifungal treatment should reduce the risk of systemic and local complications in Candida BSI. ${ }^{1}$ However, the effects of early antifungal treatment decisions on presentation of OC are incompletely understood. ${ }^{6}$

In our study, we carefully defined the properties of patients with Candida BSI who were examined or not examined by ophthalmologists due to their critical clinical condition. We investigated the role of choice and timing of early antifungal treatments on the risk of OC. We also explored various clinical features of the patients with OC in the cohort of patients with Candida BSI treated during 2008-2017. Finally, we aimed to identify risk profiles associated with $\mathrm{OC}$ and explored the detailed ocular findings of OC among our patient population.

\section{MATERIALS AND METHODS}

\section{Setting, patients and study design}

All patients (n=304) with Candida BSI during 2008-2017 treated at Oulu University Hospital responsible for tertiary care for a population of approximately 400000 inhabitants were included. Thirty-three of these patients were transferred into other hospitals for treatment or follow-up and were thus excluded from the analysis involving the OEs; the remaining 271 Candida BSI cases were evaluated. Candidemia was defined as the presence of at least one blood culture positive for Candida sp. ${ }^{19}$ Candida-positive blood culture at least 72 hours after active antifungal treatment $(\mathrm{n}=81)$ was considered persistent (median 5 days). If a patient had more than one candidemia episode, only the first episode was analysed. The study followed the tenets of the Declaration of Helsinki.

\section{Blood cultures and microbiological analysis}

Microbiological laboratory data were collected from the laboratory records. Blood culture samples were collected from the study subjects based on clinical judgement whenever BSI was suspected. Blood cultures were carried out by an automated continuous monitoring screening system of BacT/Alert and identified to species level (bio Mérieux, Marcy-l'Etoile, France). In vitro antifungal susceptibility to fluconazole, voriconazole, echinocandins and amphotericin $\mathrm{B}$ was determined with minimum inhibitory concentration gradient strip method (E-test) according to manufacturer's guidelines. Decision on number of blood cultures was based on clinical consideration (range 2-10, median 4).

\section{Candida BSI treatment guidelines}

In patients without neutropenia, Oulu University Hospital guidelines recommended during the study years administration of either empirical intravenous fluconazole $(800 \mathrm{mg}$ starting dose, followed by $400 \mathrm{mg}$ daily) or intravenous echinocandin (caspofungin or micafungin according to generally accepted dosing) whenever Candida BSI is suspected. In patients with neutropenia with suspicion of candidemia, echinocandin was recommended. For the study period, caspofungin (70 mg starting dose, followed by $50 \mathrm{mg}(<80 \mathrm{~kg}$ ) or $70 \mathrm{mg}$ $(>80 \mathrm{~kg})$ daily) has been used. Delay of at least 12 hours from the time when the first positive blood culture was drawn was recorded. Prompt removal of central venous catheters (CVCs) was recommended. The hospital guidelines recommend all patients to be considered for echocardiography (transthoracic: 185 patients, transoesophageal: 24 patients) to search for endocarditis. All patients with Candida BSI were recommended to be considered for a possibility of intraocular candidemia complications.

\section{Ophthalmological evaluation and definitions}

Comprehensive OE included measurements of visual acuity, intraocular pressure and biomicroscopy evaluations. Best corrected visual acuity measurements were converted to a logarithm of the minimum angle of resolution ( $\log M A R)$ for analysis. Some of the patients were evaluated during the consultation visit in the intensive care unit only by direct and/or indirect ophthalmoscopy and those most severely ill could not be examined due to lack of cooperation. The ocular complications of Candida BSI documented included endophthalmitis defined as vitritis or fluffy lesions extending into the vitreous body and/or chorioretinitis including focal white infiltrates restricted to the chorioretinal layers either in macular area or peripheral retina. Also, data from haemorrhages in optic disc or retina as well as macular oedema were collected.

\section{Clinical data collection and statistical analysis}

Data of patient history, previous diagnoses, foreign materials, CVCs or other foreign materials, deep infection foci and antifungal treatments were collected. The IBM SPSS Statistics V.27 was used for statistical analysis and the p value of $<0.05$ was considered as statistically significant. The $\mathrm{X}^{2}$ test was used for identifying differences between categorical variables. T-test was used for finding the differences in continuous variables between two groups.

\section{RESULTS}

\section{Patient selection and microbiological finding}

Table 1 summarises the study population $(n=304)$ divided according to the causative Candida spp. The most common blood culture findings were C. albicans $(63 \%$, $\mathrm{n}=192)$, C. glabrata $(18 \%, \mathrm{n}=54)$ and C. parapsilosis $(7 \%$, $\mathrm{n}=20)$. In addition, 38 cases of other species were identified (C. krusei $(\mathrm{n}=7)$, C. lusitaniae $(\mathrm{n}=5)$, C. guilliermondii $(\mathrm{n}=1)$, C. tropicalis $(\mathrm{n}=6)$, C. kefyr $(\mathrm{n}=1)$, C. inconspicua $(\mathrm{n}=2)$, and other yeasts including Saccharomyces cerevisae $(\mathrm{n}=11)$, Geotrichum $(\mathrm{n}=2)$, and Rhodotorula $(\mathrm{n}=3))$. There 
Table 1 Candida isolates in blood cultures

\begin{tabular}{|c|c|c|c|c|}
\hline & C. albicans & C. glabrata & C. parapsilosis & Other \\
\hline Total, n (\%) & $192(63)$ & $54(18)$ & $20(7)$ & $38(13)$ \\
\hline Male & $101(53)$ & $29(54)$ & $13(65)$ & $20(53)$ \\
\hline Female & $91(47)$ & $25(46)$ & $7(35)$ & $18(47)$ \\
\hline Mean age $\pm S D$ (years) & $61 \pm 18^{*}$ & $65 \pm 15$ & $66 \pm 13$ & $59 \pm 17$ \\
\hline Ocular examination & $73(38)$ & $16(30)$ & $6(30)$ & $8(21)$ \\
\hline Intensive care unit & $42(22)$ & $7(13)$ & $4(20)$ & 7 (18) \\
\hline Abdominal malignancy & $48(23)$ & $15(28)$ & $2(10)$ & $4(11)$ \\
\hline Leukaemia or lymphoma & $16(8)$ & $0(0)$ & $2(10)$ & $4(11)$ \\
\hline Abdominal complication & $42(22)$ & $21(39)$ & $4(20)$ & $10(27)$ \\
\hline Neurological or psychiatric & $35(18)$ & $10(19)$ & $3(15)$ & $9(24)$ \\
\hline Injury & $8(4)$ & $1(2)$ & $2(10)$ & $0(0)$ \\
\hline Alcohol abuse & $15(8)$ & $7(13)$ & $2(10)$ & 7 (18) \\
\hline Intravenous drug abuse & $8(4)$ & $0(0)$ & $1(5)$ & $5(13)$ \\
\hline Central venous catheter & $98(51)$ & $26(48)$ & $6(30)$ & $16(42)$ \\
\hline Autoimmune disease & $9(5)$ & $0(0)$ & $2(10)$ & $2(5)$ \\
\hline Endovascular or valve prosthesis & $7(4)$ & $1(2)$ & $0(0)$ & $1(3)$ \\
\hline Endocarditis & $6(3)$ & $0(0)$ & $2(10)$ & $2(5)$ \\
\hline No foreign body & $59(31)$ & $15(28)$ & $7(35)$ & $17(45)$ \\
\hline
\end{tabular}

The three most common species of Candida (C. albicans, C. glabrata, C. parapsilosis) and all other species (other) are shown. Gender and age distribution and number of patients examined by an ophthalmologist according to microbiology are presented. The underlying conditions and properties of the patients associated with the development of candidemia are also included.

${ }^{\star}$ The patients with $C$. albicans were younger than those with C. glabrata or C. parapsilosis $(p<0.05)$.

were no statistically significant differences in the patient characteristics or known risk factors for candidemia between the different Candida spp. Abdominal complications or abdominal malignancies were the most common predisposing conditions associated with the Candida BSI. About half $(n=146)$ of the patients had CVCs.

\section{Clinical features of patients examined by an ophthalmologist}

Table 2 summarises the properties of patients who were carefully examined by an experienced ophthalmologist including, for example, comprehensive $\mathrm{OE}$ by biomicroscopy $(n=103)$. The table also shows properties of patients $(n=155)$ not examined by an ophthalmologist. Thirty-three patients were excluded due to their transfer to other hospitals. Patients evaluated by direct and/ or indirect ophthalmoscopy $(\mathrm{n}=13,5 \%)$ only were also excluded. The examined patients were younger ( $57 \mathrm{vs}$ $63, \mathrm{p}<0.01)$ than those who were not examined. Patients who were treated at intensive care units $(\mathrm{p}<0.05)$ or had alcohol abuse $(p<0.01)$ were less often evaluated. Especially those who died within 3 months from the positive blood culture were rarely examined by ophthalmologists $(\mathrm{p}<0.001)$. The median time from positive blood culture to $\mathrm{OE}$ was 5.5 days (range $1-32$ days).

\section{Clinical features of patients with $\mathrm{OC}$}

Comprehensive OE was performed in 103 of the 271 (34\%) patients summarised in table 3. Thirty-five patients examined had previously been diagnosed with another eye disease such as cataract, age-related macular degeneration, open-angle glaucoma or vitreous detachment. Findings consistent with OC criteria were found in 33 of the 103 patients $(32 \%)$. Forty-six per cent of the patients with $\mathrm{OC}$ were men and the average age of all patients with OC was 55 years. Those below 40 years of age were less frequently affected by OC when compared with patients older than 40 years $(\mathrm{p}<0.05)$. C. albicans was the most common causative species in $88 \%$ of all OC cases. The features of OC caused by C. albicans or non-albicans are presented in table 4. Chorioretinitis was found in $94 \%$, endophthalmitis in $48 \%$ and both in $42 \%$ of the OC cases. A majority of the patients, $94 \%$, had bilateral involvement of OC. Macular lesion was noted in $67 \%$, macular oedema in 9\%, retinal haemorrhages in $36 \%$ and haemorrhages in optic disc in $9 \%$ of the patients with OC. Bleeding in retina and/or optic disc and endophthalmitis were mostly found in patients with $C$. albicans infection compared with OC due to other Candida spp. Vitrectomy was performed to two patients only, and three patients underwent vitreous aspiration. Intraocular pressure was at similar level in the primary and at the follow-up examinations (from $10.3 \pm 3.9$ to $11.1 \pm 4.2 \mathrm{~mm}$ $\mathrm{Hg}(\mathrm{p}=0.310)$ and from $10.7 \pm 4.1$ to $11.4 \pm 3.7 \mathrm{~mm} \mathrm{Hg}$ $(\mathrm{p}=0.463)$ in right and left eyes, respectively). The visual acuity improved from $0.36 \log$ MAR to $0.25 \log$ MAR when compared between the acute phase and the follow-up visit $(\mathrm{p}=0.003)$. Other clinical parameters (underlying 
Table 2 Properties of patients examined or not examined by an ophthalmologist

\begin{tabular}{lll}
\hline & \multicolumn{2}{l}{ Ophthalmological examination } \\
\cline { 2 - 3 } & Yes & No \\
\hline Total, $\mathrm{n}(\%)$ & 103 & 155 \\
Male & $56(54)$ & $84(54)$ \\
\hline Female & $47(46)$ & $71(46)$ \\
\hline Mean age \pm SD (years) & $57 \pm 18^{\star}$ & $63 \pm 17$ \\
\hline Intensive care unit & $13(13) \dagger$ & $42(27)$ \\
\hline Abdominal malignancy & $31(30)$ & $33(21)$ \\
\hline Leukaemia or lymphoma & $5(5)$ & $15(10)$ \\
\hline Abdominal complication & $30(29)$ & $43(28)$ \\
\hline Neurological or psychiatric & $20(19)$ & $32(21)$ \\
\hline Injury & $4(4)$ & $6(4)$ \\
\hline Alcohol abuse & $4(4)^{\star}$ & $26(17)$ \\
\hline Intravenous drug & $8(8)$ & $6(4)$ \\
\hline Central venous catheter & $58(54)$ & $79(51)$ \\
\hline Autoimmune disease & $3(3)$ & $9(6)$ \\
\hline Endovascular or valve & $3(3)$ & $5(3)$ \\
prosthesis & & $4(3)$ \\
\hline Endocarditis & $5(5)$ & $55(35)$ \\
\hline Death <3 months & $3(3) \ddagger$ & \\
\hline
\end{tabular}

Age and gender distribution are shown. In addition, conditions and properties associated with development of candidemia are presented. Those treated at intensive care units, patients suffering from alcohol-related complications or died within 3 months were less frequently examined by an ophthalmologist.

${ }^{*} \mathrm{P}<0.01$.

$\dagger P=0.05$.

$\ddagger \mathrm{P}<0.001$

disease, foreign body involvement and survival) for patients with $\mathrm{OC}$ are presented in table 3. More than half of the patients with OC, $58 \%$, survived despite the concurred Candida BSI. There was no statistically significant difference in the mortality rate of the patients with Candida BSI with or without OC.

\section{Effect of antifungal treatments on risk of $\mathrm{OC}$}

We explored the possibility that this choice of empirical antifungal treatment may affect development of OC (table 5). We also considered the possibility that the properties of the patients presented in tables 1 and 2 would associate with the risk of developing OC. We found that those with the most common underlying properties, CVC, or abdominal malignancy, appeared to have lower risk of OC when they were treated with echinocandin compared with those who received fluconazole $(\mathrm{p}=0.01)$. We also found that none of the patients without C. albicans who received echinocandin treatment developed OC.

Delay in antifungal treatment

We also evaluated the effect of delay in initiation of antifungal treatment on the risk of OC calculated from the time when the positive blood culture was drawn.
Table 3 A total of 103 patients were examined by an ophthalmologist

\begin{tabular}{|c|c|c|}
\hline & \multicolumn{2}{|c|}{ Ocular candidiasis } \\
\hline & Yes & No \\
\hline Total, n (\%) & $33(32)$ & $70(68)$ \\
\hline Male & $15(46)$ & $41(59)$ \\
\hline Female & $18(55)$ & $29(41)$ \\
\hline Mean age $\pm S D$ (years) & $55 \pm 13$ & $61 \pm 19$ \\
\hline Candida albicans & $29(88)^{*}$ & $44(63)$ \\
\hline C. glabrata & $2(6)$ & $14(20)$ \\
\hline C. parapsilosis & $0(0)$ & $6(9)$ \\
\hline Other Candida spp & $2(6)$ & $6(9)$ \\
\hline No persisting blood culture ( $>72$ hours) & $16(48)$ & $49(70) \dagger$ \\
\hline Intensive care unit & $4(12)$ & $9(13)$ \\
\hline Abdominal malignancy & $14(42)$ & $17(24)$ \\
\hline Leukaemia or lymphoma & $4(12)$ & $1(1)$ \\
\hline Neutropenia $\left(<0.1 \times 10^{9} / \mathrm{L}\right)$ & $3(9)$ & $2(3)$ \\
\hline Abdominal complication & $6(18)$ & $24(34)$ \\
\hline Neurological or psychiatric & $4(12)$ & $16(23)$ \\
\hline Injury & $1(3)$ & $3(4)$ \\
\hline Alcohol abuse & $1(3)$ & $3(4)$ \\
\hline Intravenous drug & $1(3)$ & $7(10)$ \\
\hline Central venous catheter (CVC) & $21(64)$ & $37(53)$ \\
\hline CVC removal confirmed $\neq$ & $15(71)$ & $28(76)$ \\
\hline Autoimmune disease & $1(3)$ & $2(3)$ \\
\hline Endovascular or valve prosthesis & $2(6)$ & $1(1)$ \\
\hline Endocarditis & $0(0)$ & $5(7)$ \\
\hline Death $<3$ months & $2(6)$ & $1(1)$ \\
\hline Alive $>1$ year & $19(58)$ & $40(57)$ \\
\hline
\end{tabular}

The table shows properties of those who had ocular candidiasis $(n=33)$ compared with patients negative for ocular candidiasis $(n=70)$. Conditions and properties related with development of candidemia are shown. Patients with C. albicans were more frequently affected. Patients in whom blood cultures were negative for candida during antifungal treatment (72 hours) had a lower number of ocular candidiasis.

${ }^{*} \mathrm{P}<0.01$.

$+\mathrm{P}=0.05$

$\ddagger$ Prompt removal of CVC was documented in 43 (74\%) of 58 patient files.

Table 4 The table summarises the findings in patients with ocular candidiasis $(\mathrm{OC})$

\begin{tabular}{ll}
\hline & All Candida \\
\hline Bilateral OC & $\mathbf{n = 3 3}$ \\
\hline Chorioretinitis & $31(94)$ \\
Endophthalmitis & $31(94)$ \\
Chorioretinitis and endophthalmitis & $16(48)$ \\
Macula affected & $14(42)$ \\
Haemorrhages & $22(67)$ \\
\hline
\end{tabular}


Table 5 Effect of the timely empirical antifungal treatment $(<12$ hours) or delayed antifungal treatment $(>12$ hours) on positive $(\mathrm{OC}+)$ or negative $(\mathrm{OC}-)$ ocular candidiasis finding in relation with fluconazole or echinocandin treatment. In addition, association of the most common risk factors (presence of central venous catheter or abdominal malignancy) with positive or negative OC finding in relation with fluconazole or echinocandin treatment

\begin{tabular}{|c|c|c|c|c|}
\hline \multirow{2}{*}{$\begin{array}{l}\text { Antifungal treatment } \\
\text { OC }\end{array}$} & \multicolumn{2}{|l|}{$\begin{array}{l}\text { Fluconazole } \\
\text { n (\%) }\end{array}$} & \multicolumn{2}{|c|}{$\begin{array}{l}\text { Echinocandin } \\
\text { n (\%) }\end{array}$} \\
\hline & $\mathrm{OC}+$ & OC- & $\mathrm{OC}+$ & OC- \\
\hline All Candida $(\mathrm{n}=101)$ & $20(19.8)$ & $29(28.7)$ & $12(11.8)$ & 40 (39.6) \\
\hline Treatment delay $<12$ hours $(n=68)$ & $14(20.5)$ & $18(26.4)$ & $9(13.2)$ & $27(39.7)$ \\
\hline Treatment delay $>12$ hours $(n=29)$ & $4(13.7)$ & $9(31.0)$ & $3(10.3)$ & $13(44.8)$ \\
\hline Central venous catheter $(n=57)$ & $15(26.3)$ & $14(24.5)$ & $6(10.5)^{\star}$ & $22(38.6)$ \\
\hline Abdominal malignancy $(n=31)$ & $11(35.4)$ & 7 (22.6) & $2(6.5)^{*}$ & $10(32.2)$ \\
\hline Candida albicans $(\mathrm{n}=73)$ & $17(23.2)$ & $16(21.9)$ & $12(16.4)$ & $28(38.3)$ \\
\hline Treatment delay $<12$ hours $(n=48)$ & $12(25.0)$ & $9(18.8)$ & $9(18.8)$ & $18(37.6)$ \\
\hline Treatment delay $>12$ hours $(n=22)$ & $3(13.6)$ & $6(27.2)$ & $3(13.6)$ & $10(45.4)$ \\
\hline Central venous catheter $(n=43)$ & $15(34.9)$ & 7 (16.2) & $6(13.9)^{*}$ & $15(34.9)$ \\
\hline Abdominal malignancy $(\mathrm{n}=21)$ & 9 (42.9) & $4(19.0)$ & $2(9.5)$ & $6(28.6)$ \\
\hline Non-albicans $(\mathrm{n}=28)$ & $3(10.7)$ & $13(46.4)$ & $0(0)$ & $12(42.9)$ \\
\hline Treatment delay $<12$ hours $(n=20)$ & $2(10.0)$ & $9(45.0)$ & $0(0)$ & $9(45.0)$ \\
\hline Treatment delay $>12$ hours $(n=7)$ & $1(14.2)$ & $3(42.9)$ & $0(0)$ & $3(42.9)$ \\
\hline Central venous catheter $(n=15)$ & $1(6.7)$ & $7(46.7)$ & $0(0)$ & $7(46.7)$ \\
\hline Abdominal malignancy $(n=9)$ & $2(22.2)$ & $3(33.3)$ & $0(0)$ & $4(44.4)$ \\
\hline
\end{tabular}

${ }^{*} \mathrm{P}<0.05$.

However, no statistical difference in the number of OC was seen when we compared all cases with no delay with those who experienced a delay of 12 hours or longer in their antifungal treatment. In contrary, a significant benefit of early antifungal medication in the number of endophthalmitis was observed; those who received any antifungal medication within 12 hours had a lower number of endophthalmitis $(\mathrm{n}=14)$ when compared with cases with a delay of more than 12 hours $(n=4, p<0.01)$.

\section{Blood cultures during antifungal treatment}

We looked for the possibility that patients in whom blood culture positivity persisted during antifungal treatment would be at higher risk of developing OC. We found that patients in whom blood cultures remained positive for C. albicans during antifungal treatment were at elevated risk of developing $\mathrm{OC}(\mathrm{p}=0.02)$. These patients had more frequently CVC $(\mathrm{p}<0.001)$. We also observed that the $C$. albicans-positive patients who did not have CVC and who received early echinocandin treatment were less likely to have a persistent blood culture finding $(\mathrm{p}=0.05)$.

\section{Endocarditis and $\mathrm{OC}$}

In our cohort, five cases of endocarditis with C. albicanspositive blood culture were confirmed and none of them developed OC. All these cases occurred in association with intravenous drug abuse. Mean age of patients with drug addiction was significantly lower (32 years vs 65 years, $\mathrm{p}<0.001)$ compared with the whole study population.

\section{DISCUSSION}

The incidence of OC in candidemia may range from $3 \%$ to $27 \% .{ }^{10}$ Ophthalmological consultation is recommended to all patients with candidemia ${ }^{1-35}$ although some studies have failed to provide evidence supporting routinely performed ocular assessment. ${ }^{1411}$ Patients with candidemia are often severely ill and ophthalmological analysis may not be possible; in our study, OE was completed to the selected patients due to their critical condition. The examined patients appeared to be of younger age and healthier compared with those who were not examined (table 2). Patients at intensive care units, and those with alcohol-related conditions or poor prognosis, for example, were less frequently examined. Finally, 32\% of the comprehensively examined patients in our study had ocular findings consistent with OC. It must be appreciated that the observed selection bias may have influenced our results. We believe that previous OC investigations are also based on selected patient material.

In agreement with the previous studies, the leading fungal isolate $(88 \%)$ among our patients with $\mathrm{OC}$ was $C$. albicans. ${ }^{3512}$ We also found that those patients in whom blood cultures remained positive for C. albicans during antifungal treatment were at OC risk. Importantly, a shorter duration in persistence of candidemia was seen in echinocandin-treated patients with CVC. It is known that echinocandins can be active against Candida biofilm. ${ }^{13}$ Evidence supporting the possibility that echinocandins can be more rapid in clearance of candidemia compared 
with triazoles has also been presented. ${ }^{14}$ It seems possible that efficient clearance of candidemia and activity against Candida biofilm may protect from developing eye complications in candidemia.

Early treatment with any antifungal medication reduced the number of endophthalmitis; those who received either fluconazole or echinocandin within 12 hours had a lower number of endophthalmitis when compared with cases with a delay of more than 12 hours $(p<0.01)$. However, a delay in antifungal treatment was not associated with an increase in chorioretinitis when the entire study population was considered. In addition, no advantage of fluconazole, a drug of choice in intraocular C. albicans infections, was seen. First-line use of echinocandin with low intraocular concentration appeared favourable compared with fluconazole at least in patients with CVC or abdominal malignancy. Interestingly, none of the patients without C. albicans who received echinocandin developed OC. This is in line with the recent study that reported no increased ocular involvement in patients with Candida BSI initially treated with echinocandins. ${ }^{15}$

Lack of CVC in association with echinocandin treatment protected from persistent candidemia in our study. This result highlights the importance of prompt CVC removal in candidemia. It is also known that persisting blood cultures can be seen in endocarditis. ${ }^{16}{ }^{17}$ Although we completed echocardiography to a high proportion of our patients, none of the patients with endocarditis with C. albicans BSI developed OC. It seems possible that the young age of our patients with endocarditis protected them from OC. This is supported by the fact that risk of OC in our study was significantly lower in those below 40 years of age compared with the whole patient population. Apparently, routine echocardiography may not be necessary to identify those at OC risk. The ophthalmological assessment for OC might thus be justified by the overall risk profile of a patient.

Early appropriate antimicrobial treatment improves prognosis of patients with Candida BSI. ${ }^{18-20}$ In our study, an early initiation of antifungal medication reduced the risk of endophthalmitis. Time between the positive blood culture and initial OE can affect the OC rate. ${ }^{321}$ Diagnostic findings in endophthalmitis, for example, may require several days to develop. ${ }^{10}$ Ophthalmological methods and extent of evaluation can be variable in studies involving $\mathrm{OC}$ in candidemia. Cooperation required for reliable $\mathrm{OE}$ may not be possible in intensive care settings; priority must be given to life-saving treatments in critical conditions. In conclusion, the selection of patients referred to ophthalmologists, timing of $\mathrm{OE}$ and the used methods may affect the reported frequencies.

In summary, our study based on careful ocular biomicroscopy analysis of patients with candidemia provides evidence supports the view that choice and timing of the first-line antifungal treatment affects the risk of ocular complications. It must be noted, however, that we evaluated the possibility of preventing OC by the choice of antifungal medication. Our results, however, do not provide information on the optimal OC treatment. Still, early treatment with any antifungal agent and echinocandin treatment in selected cases may protect the patients with candidemia from developing OC.

Contributors $\mathrm{NMH}$ and $\mathrm{TH}$ - study design, data collection, analysis and manuscript preparation. HK and JS—data collection and analysis. MS—study design and manuscript preparation.

Funding The authors have not declared a specific grant for this research from any funding agency in the public, commercial or not-for-profit sectors.

Competing interests None declared.

Patient and public involvement Patients and/or the public were not involved in the design, or conduct, or reporting, or dissemination plans of this research.

Patient consent for publication Not applicable.

Ethics approval Approved by Oulu University Hospital Ethics Committee (89/2017). Provenance and peer review Not commissioned; externally peer reviewed.

Data availability statement Data are available upon reasonable request. Available upon request.

Open access This is an open access article distributed in accordance with the Creative Commons Attribution Non Commercial (CC BY-NC 4.0) license, which permits others to distribute, remix, adapt, build upon this work non-commercially, and license their derivative works on different terms, provided the original work is properly cited, appropriate credit is given, any changes made indicated, and the use is non-commercial. See: http://creativecommons.org/licenses/by-nc/4.0/.

ORCID iDs

Nina Hautala http://orcid.org/0000-0001-5454-5602

Timo Hautala http://orcid.org/0000-0001-7889-1614

\section{REFERENCES}

1 Pappas PG, Kauffman CA, Andes DR, et al. Executive summary: clinical practice guideline for the management of candidiasis: 2016 update by the infectious diseases Society of America. Clin Infect Dis 2016;62:409-17.

2 Krishna R, Amuh D, Lowder CY, et al. Should all patients with candidaemia have an ophthalmic examination to rule out ocular candidiasis? Eye 2000;14 (Pt 1):30-4.

3 Ueda T, Takesue Y, Tokimatsu I, et al. The incidence of endophthalmitis or macular involvement and the necessity of a routine ophthalmic examination in patients with candidemia. PLoS One 2019;14:e0216956.

4 El-Abiary M, Jones B, Williams G, et al. Fundoscopy screening for intraocular Candida in patients with positive blood cultures-is it justified? Eye 2018;32:1697-702.

5 Son $\mathrm{H}-J$, Kim MJ, Lee S, et al. Risk factors and outcomes of patients with ocular involvement of candidemia. PLoS One 2019;14:e0222356.

6 Breazzano MP, Day HR, Bloch KC, et al. Utility of ophthalmologic screening for patients with Candida bloodstream infections: a systematic review. JAMA Ophthalmol 2019;137:698-710.

7 Kullberg BJ, Arendrup MC. Invasive candidiasis. N Engl J Med 2015;373:1445-56

8 Felton T, Troke PF, Hope WW. Tissue penetration of antifungal agents. Clin Microbiol Rev 2014;27:68-88.

9 Vinikoor MJ, Zoghby J, Cohen KL, et al. Do all candidemic patients need an ophthalmic examination? Int J Infect Dis 2013;17:e146-8.

10 Nagao M, Saito T, Doi S, et al. Clinical characteristics and risk factors of ocular candidiasis. Diagn Microbiol Infect Dis 2012;73:149-52.

11 Vena A, Muñoz P, Padilla B, et al. Is routine ophthalmoscopy really necessary in candidemic patients? PLoS One 2017;12:e0183485

12 Oude Lashof AML, Rothova A, Sobel JD, et al. Ocular manifestations of candidemia. Clin Infect Dis 2011;53:262-8.

13 Ghannoum M, Roilides E, Katragkou A, et al. The role of echinocandins in Candida Biofilm-Related vascular catheter infections: in vitro and in vivo model systems. Clin Infect Dis 2015;61 Suppl 6:S618-21.

14 Lin K-Y, Chen P-Y, Chuang Y-C, et al. Effectiveness of echinocandins versus fluconazole for treatment of persistent candidemia: a timedependent analysis. J Infect 2018;77:242-8. 
15 Muñoz P, Vena A, Padilla B, et al. No evidence of increased ocular involvement in candidemic patients initially treated with echinocandins. Diagn Microbiol Infect Dis 2017;88:141-4.

16 Pierce D, Calkins BC, Thornton K. Infectious endocarditis: diagnosis and treatment. Am Fam Physician 2012;85:981-6.

17 Gupta A, Mendez MD. Endocarditis. In: StatPearls. Treasure Island (FL): StatPearls Publishing, 2020.

18 Garey KW, Rege M, Pai MP, et al. Time to initiation of fluconazole therapy impacts mortality in patients with candidemia: a multiinstitutional study. Clin Infect Dis 2006;43:25-31.
19 Farmakiotis D, Kyvernitakis A, Tarrand JJ, et al. Early initiation of appropriate treatment is associated with increased survival in cancer patients with Candida glabrata fungaemia: a potential benefit from infectious disease consultation. Clin Microbiol Infect 2015;21:79-86.

20 Morrell M, Fraser VJ, Kollef MH. Delaying the empiric treatment of Candida bloodstream infection until positive blood culture results are obtained: a potential risk factor for hospital mortality. Antimicrob Agents Chemother 2005;49:3640-5.

21 Donahue SP, Greven CM, Zuravleff JJ, et al. Intraocular candidiasis in patients with candidemia. Clinical implications derived from a prospective multicenter study. Ophthalmology 1994;101:1302-9. 